\title{
Analysis of fractal events with background
}

\author{
Tatiana Dedovich ${ }^{1, \star}$ and Mikhail Tokarev ${ }^{1, \star \star}$ \\ ${ }^{1}$ Joint Institute for Nuclear Research, Dubna, Russia
}

\begin{abstract}
Properties of self-similarity and fractality in the processes of interactions of hadrons and nuclei at high energies are discussed. Different methods of fractal analysis (the box counting $B C$, system of the equations of p-adic coverage $\mathrm{SePaC}$ methods) are described. Fractal analysis of mixed events was carried out by $B C$ and $\mathrm{SePaC}$ methods. The procedure of separation of fractals and background, estimation of the number of fractals in the original data set and contamination of the extracted data was proposed. The dependence of event contamination on multiplicity and background is studied. Reconstruction of the spectrum of fractal dimensions is found to depend on the method and background.
\end{abstract}

\section{Introduction}

The concept of the fractal is widely used in high-energy physics. Among them there are the fractal solution for the Schrödinger equation [1-3], the fractal space-time as a new method of regularization in the quantum field theory [4-7], the topological charge density distribution in the lattice gluodynamics as fractals [8], the intermittency in the spectra of secondary particles [9-11], the fractal structures of thermodynamical functions [12], the fractality of the momentum space as a signal of criticality in nuclear collisions [13], and the fractal properties of nuclei and events [14, 15].

A fractal is defined as a self-similar object characterized by the fractal dimension $D_{F}$ which is more than the topological one $D_{T}$ for the same object. The topological dimension determines the measure $M=\lim _{l \rightarrow 0} \sum_{i=1}^{N} l^{D_{T}}$, where $N$ is a number of probes, covering the object with a size $l$. For the euclidean object this measure is independent of the scale, has a finite limit and determines the size of the object (length, square, volume). For a fractal the measure $M$ tends to infinity, because $N$ increases faster than a size $l$ is reduced. In order to evaluate the rate of the growth the Hausdorff measure $M_{H}=\lim _{\delta \rightarrow 0} \sum_{i=1}^{N} l_{i}^{d}$ is introduced. Here $N$ is a number of probes with a size $l<\delta$. The fractal dimension $D_{F}$ is the value of $d$ which provides the finite value of the measure $M_{H}$. Every fractal is characterized by the fractal dimension $D_{F}$ and the box dimension $D_{b}=\lim _{\delta \rightarrow 0} \ln N / \ln l$. There are various types of fractals. Among them are fractals with independent, dependent and combined partition. They are analysed by the $\mathrm{SePaC}, \mathrm{BC}$ and $\mathrm{PaC}$ methods in [16-18]. It was found, in particular, that $D_{F}$ and $D_{b}$ can be different for some types of fractals.

\footnotetext{
$\star^{\star}$ e-mail: tdedovich@yandex.ru

$\star \star$ e-mail: tokarev@jinr.ru
} 


\section{2 z-Scaling}

The description of inclusive cross sections of hadron production in collisions of protons and nuclei at high energies based on the principles of locality, self-similarity, fractality in the framework of the $z$-scaling concept was proposed in [19]. Authors have concluded that the structure of hadrons, mechanism of constituent interactions and hadronization process reveal self-similar properties over a wide scale range. In this approach an inclusive cross section is described in terms of the dimensionless function $\psi(z)$ depending on the self-similarity parameter $z$. The fractality is reflected in definition of $z=z_{0}\left(1-x_{1}\right)^{-\delta_{1}}\left(1-x_{2}\right)^{-\delta_{2}}$. Here $z_{0}$ is a slowly changing function of the kinematic variables, $x_{1}$ and $x_{2}$ are the scale-invariant fractions of incoming four-momenta $P_{1}, P_{2}$ of colliding objects and $\delta_{1}, \delta_{2}$ are their fractal dimensions. This methods has been applied for analysis of the inclusive spectra of hadrons and jets production at Tevatron and LHC [20,21]. The dependence of the scaling function $\psi$ on $z$ was found to be independent of the collision energy and angle of produced particle. It is also described by the power law $\psi(z) \sim z^{-\beta}$ at high $z$. The value of the slope parameter $\beta$ is independent of kinematic variables over a wide $z$-range. It confirms self-similarity and fractality of hadron production at various scales. Discontinuity of dimensions $\delta_{1}, \delta_{2}$ is assumed to be an indication of phase transition which is related to the change of physical sub-processes underlying inclusive particles production. Fractal dimensions have been determined from data analysis of inclusive cross sections and then verified in analysis of other data sets. These dimensions are fundamental characteristics of particles and processes and should be determined from the fractal analysis of the experimental data.

\section{Fractal analysis}

The fractal analysis is aimed to determine distributions characterizing the fractal properties of particles and processes. First of all the phase space in which the object reveals its fractal properties and an adequate method of analysis should be found. The original data set should be also described.

In the present work we use $B C$ [16] and $S e P a C$ [18] methods to study mixed events $N_{e v}=$ $N_{\text {frac }}+N_{b g}$ consisting of fractals $N_{f r a c}$ and background $N_{b g}$. The fractals $\left(N_{f r a c}=1857\right)$ of various types are generated by using the procedure of independent, dependent and combined partition. The background is chosen as to be random data set. Portion of background is changed from 0.05 to 0.95 . The preliminary analysis includes estimation of number of fractals and extraction of data set of reconstructed fractals. Further the extracted data set is analysed to determine of spectrum of fractal dimensions and contamination.

\section{$4 B C$ method}

In the section we briefly describe the $B C$ method [16]. It consists of the following steps:

1. Reading out the data $X$.

2. Construction of the set of distributions of $X$. The number of bins $M_{i}$ in distributions changes as multiplicatively $M_{i}=(P)^{i}$ or additively $M_{i+1}=M_{i}+$ step .

3. Counting the number of non-zero $N$ bins for each distribution.

4. Finding the value of the slope parameter $D_{b}$ of the power function $N \sim M^{D_{F}}$ and corresponding $\chi^{2}$ of the linear approximation of this function in a double-log scale.

6. Verification of the accuracy condition: if $\chi^{2}<\chi_{\text {lim }}^{2}$ then the set of particles is considered to be a fractal.

Notice, that the $B C$ method determines box dimension $D_{b}$ and has one parameter $\chi_{\text {lim }}^{2}$. The dimension errors are strongly dependent of the set of distributions and region of linear approximation. 
Further we present the results of analysis of various events. The dependence of the portion Prtn of the events reconstructed as fractals on $\chi^{2}$ for fractal, background and mixed events with different multiplicity $N=64,27$ is shown in figure 1 .

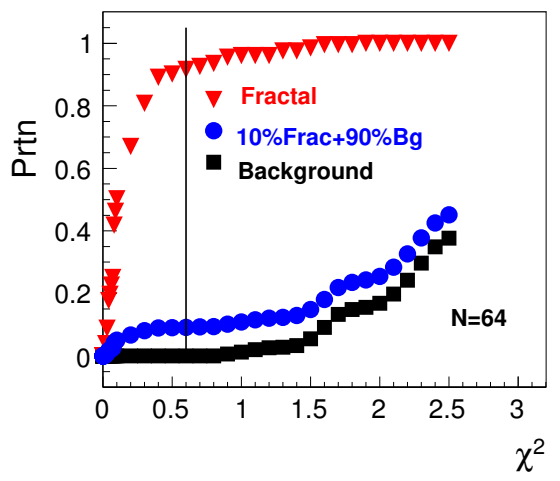

(a)

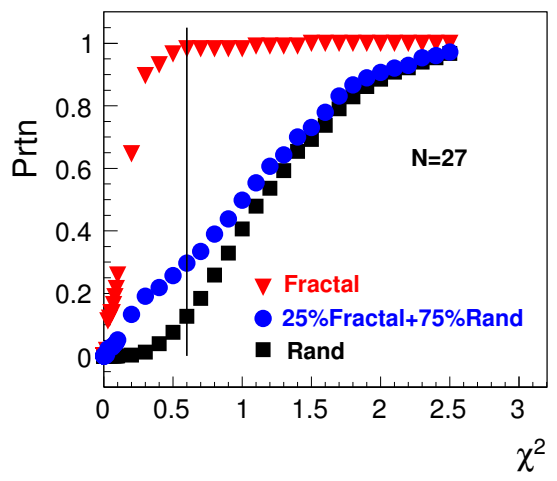

(b)

Figure 1. $B C$ : the dependence of the portion Prtn of the events reconstructed as fractals on $\chi_{\text {lim }}^{2}$ for fractal, background and mixed events with different multiplicity $N=64$ (a), $N=27$ (b).

One can see from figure 1 that for fractals (red symbols) the value of min $\chi^{2}$ at which Prtn $\approx 0.01$ does not exceed 0.1 . The portion of all fractals which are reconstructed as fractals at $\chi^{2}=0.6$ reaches 0.9-0.96. For background (black symbols) the value of min $\chi^{2}$ increases and Prtn at $\chi^{2}=0.6$ decreases with increasing $N$. Notice, that fractals and background with multiplicity $N=8$ are found to be not separable. The condition $\min \chi^{2} \leq 0.1$ corresponds to the existence of fractals in the original data set. The value 0.6 of $\chi^{2}$ was used to extract fractals from the mixed events. We found that the extracted data $N_{e x t}=P_{\text {frac }} \cdot N_{\text {frac }}+P_{b g} \cdot N_{b g}$ contain a significant portion of fractals $\left(P_{\text {frac }}=0.9 \div 0.98\right)$. For multiplicity $N \geq 64$ there is no background $\left(P_{b g}=0\right)$. For other multiplicities the extracted data have some portion $\left(P_{b g} \neq 0\right)$ of the background. Thus, we can estimate $N_{f r a c}$ in the original data set and contamination Cont of the extracted data as follows:

$$
N_{\text {frac }}=\frac{N_{e x t}-P_{b g} \cdot N_{e v}}{P_{\text {frac }}-P_{b g}}, \quad \text { Cont }=\frac{P_{b g}}{N_{e x t}} \cdot \frac{P_{f r a c} \cdot N_{e v}-N_{e x t}}{P_{\text {frac }}-P_{b g}} .
$$

\section{$5 \mathrm{SePaC}$ method}

In the section we briefly describe the $\mathrm{SePaC}$ method [18]. It consists of the following steps:

1. Reading out the data $X$.

2. Construction of $P$-adic coverages: $P=3, . . P_{\text {Max }}$. Each coverage is a set of distributions of variable

$X$. The number of bins $M_{i}$ in distributions changes as a degree of basis $P: M_{i}=(P)^{i}$.

3. Counting the number of non-zero bins $N(l e v, P)$.

4. Analysis of the system of the equations for verification of hypothesis of independent/dependent partition:

a. Construction of the system of the equations for all levels: $\sum_{i=1}^{N_{l e v}}\left(d_{l e v}\right)^{D_{F}^{l e v}}=1$. Here $N_{l e v}$ is a number of levels, and $d_{\text {lev }}$ is the length of non-zero ranges for each level.

b. Finding the solution $D_{F}^{l e v}$ of equations for each level by using the dichotomy method. 
c. Determination of the averaged value $\left\langle D_{F}^{\text {lev }}\right\rangle$ and deviation $\Delta D_{F}^{l e v}$.

d. Verification of the accuracy condition: if $\Delta D_{F}^{l e v}<D e v$, then the set of particles is considered to be a fractal ( $P$ is the base and $D_{F}=<D_{F}^{l e v}>$ is the fractal dimension).

Notice, that the $\mathrm{SePaC}$ method determines fractal dimension $D_{F}$ and has two parameters: maximal base $P_{\text {Max }}$ and deviation Dev.

Further we present the results of analysis of various events. The dependence of the portion Prtn of the events reconstructed as fractals on $P_{M a x}$ at $D e v=0.18$ for fractal, background and mixed events with different multiplicity $N=64,27$ is shown in figure 2 . One can see from figure 2 , that for

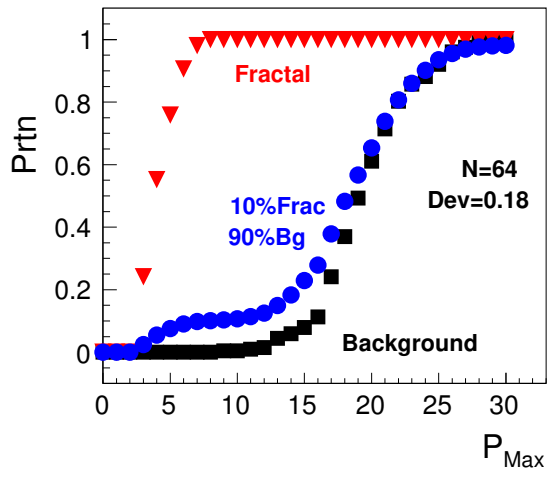

(a)

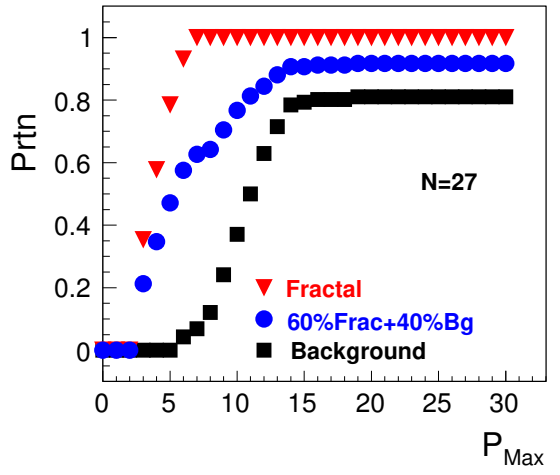

(b)

Figure 2. SePaC: the dependence of the portion Prtn of the events reconstructed as fractal on Dev for fractal, background and mixed events with different multiplicity $N=64$ (a), $N=27$ (b).

fractals (red symbols) the value $\min P_{M a x}$ at which $\operatorname{Prtn} \neq 0$ is equal to 3 . Full reconstruction of the fractals takes place when $P_{M a x}$ is equal to the minimal value on the plateau. For background (black symbols) the value of $\min P_{\text {Max }}$ increases with increasing $N$. The condition $\min P_{\text {Max }}=3$ corresponds to the existence of fractals in the original data set. The difference in the values of $\min P_{\text {Max }}$ for fractals and background was used to extract fractals from the mixed events. The value of $P_{\text {Max }}$ is taken to be minimal value at the first plateau or the value on the shoulder. We found that the extracted data $N_{e x t}=N_{f r a c}+P_{b g} \cdot N_{b g}$ contain all fractals. For multiplicity $N \geq 64$ there are not background $\left(P_{b g}=0\right)$. For other multiplicities the extracted data have some portion $\left(P_{b g} \neq 0\right)$ of the background. Thus, we can determine $N_{\text {frac }}$ in the original data set and contamination Cont of the extracted data as follows:

$$
N_{\text {frac }}=\frac{N_{e x t}-P_{b g} \cdot N_{e v}}{1-P_{b g}}, \quad \text { Cont }=\frac{P_{b g}}{N_{e x t}} \cdot \frac{P_{f r a c} \cdot N_{e v}-N_{e x t}}{1-P_{b g}} .
$$

\section{Fractal dimension}

We found that the $\mathrm{SePaC}$-method separates the fractals and the background for all multiplicities and $B C$ - only for events with $N \geq 16$. For high multiplicity events $(N \geq 64)$ both methods completely suppress background. For events with smaller multiplicities contamination Cont is found to be approximately equal for both methods. For mixed events with multiplicity $N=32,27$ and background less than $75 \%$ the value of Cont for extracted data is estimated to be less than $30 \%$. The similar result is obtained for events with $N=16,8$ and background less than $50 \%$. 
We would like to notice that the distribution of fractal dimension $D_{F}$ for the extracted data is distorted due to systematic errors of the method and the presence of background events. Figure 3 shows the distribution of the error $E r_{D_{F}}$ of fractal dimension found by the $\mathrm{SePaC}$ and $B C$ methods for fractals with independent, dependent and combined partition.

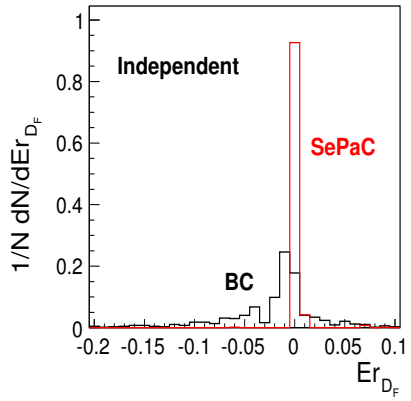

(a)

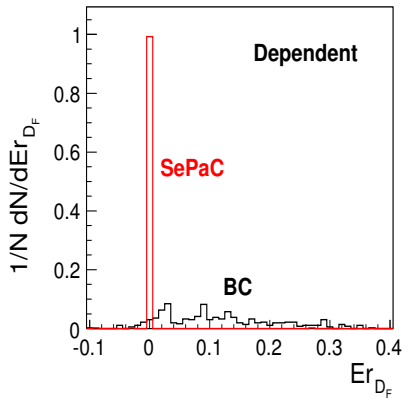

(b)

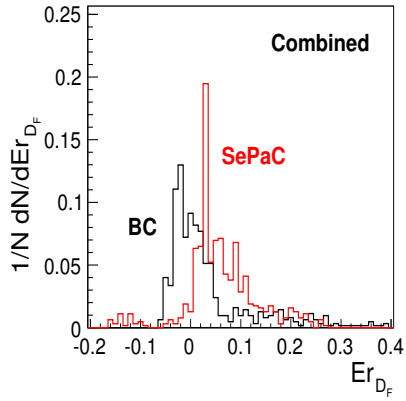

(c)

Figure 3. The distribution of the error $E_{D_{F}}$ of fractal dimension found by $\mathrm{SePaC}$ (red line) and $B C$ (black line) methods for fractals with independent (a), dependent (b) and combine (c) partition.

One can see from figure 3, that the $\mathrm{SePaC}$ method exactly reconstructs dimension $D_{F}$ for fractals with independent and dependent partition. For fractals with combined partition the distribution is found to be wide and shifted to positive values of $E r_{D_{F}}$. The distributions of errors obtained by the $B C$ method demonstrate the asymmetric shape for all types of fractals. They are shifted to negative (a), positive (b) values of $E r_{D_{F}}$ for fractals with independent and dependent partition, respectively. For fractals with combined partition the distribution (c) has positive and negative values of $E r_{D_{F}}$.

The distribution of fractal dimension $D_{F}$ for background events with different multiplicity is shown in figure 4.

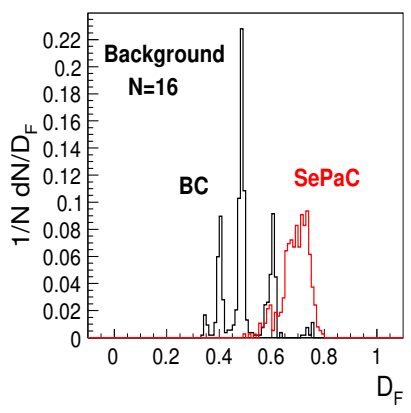

(a)

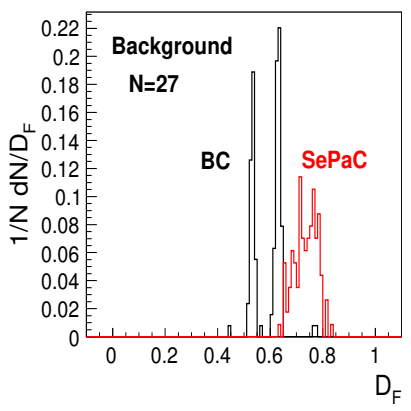

(b)

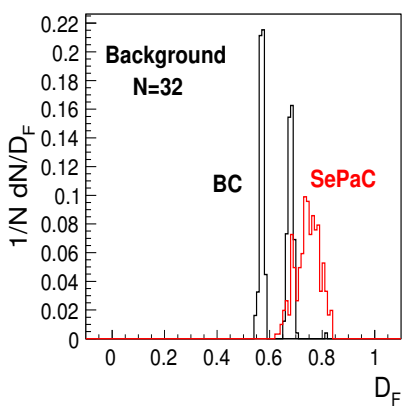

(c)

Figure 4. The distribution of fractal dimension $D_{F}$ for background events with multiplicity $N=16$ (a), 27 (b), 32 (c).

As it is seen from figure 4 the spectra of fractal dimensions reconstructed by the $B C$ and $S e P a C$ methods from the background events are different. Notice that $D_{F}$ changes in the range 0.6-0.8 for distribution obtained by the $\mathrm{SePaC}$ method. The spectrum of $D_{F}$ reconstructed by the $B C$ method has a more complex structure. 


\section{Conclusions}

Fractal analysis of mixed events was carried out by the $B C$ and $\mathrm{SePaC}$ methods. The procedure of separation of fractals and background, estimation of the number of fractals $\left(N_{\text {frac }}\right)$ in the original data set and contamination (Cont) in the extracted data was proposed. We found that the $S e P a C$-method separates the fractals and the background for all multiplicities and $B C$ - only for events with $N \geq 16$. For high multiplicity events $(N \geq 64)$ both methods completely suppress background. For events with smaller multiplicities contamination Cont was found to be approximately equal for both methods. The value of Cont depends on event multiplicity and background. It was shown that the distribution of fractal dimension $D_{F}$ for the extracted data is distorted due to systematic errors of the used method and the presence of background events.

\section{References}

[1] M.V. Berry, J.Phys.A: Math.Gen. 29, 6617 (1996)

[2] D. Wojcik et al., Phys.Rev.Lett. 85, 5022 (2000)

[3] E. Akkermans et al., Phys.Rev.Lett. 105, 230407 (2010)

[4] K. Svozil, J. Phys. A: Math. Gen. 20, 3861

[5] A. Kar, S. Rajeev, Annals Phys. 327, 102 (2012)

[6] G. Calcagni, Phys.Rev. D 84, 061501 (2011)

[7] D. Moore et al., Phys.Rev. D 90, 024075 (2014)

[8] P. Buividovich et al., Phys.Rev. D 86, 074511 (2012)

[9] I. Dremin, Surveys High Energ.Phys. 6, 141 (1992)

[10] M.K. Ghosh et al., DAE Symp.Nucl.Phys. 54, 590 (2009)

[11] M. Rasool, S. Ahmad, Chaos Solitons Fractals 84, 58 (2016)

[12] A. Deppman, Phys.Rev. D 93, 054001 (2016)

[13] N.G. Antoniou, Phys.Rev. C 93, 014908 (2016)

[14] Wei-Hu Ma et al., Chin.Phys. C 39, 104101 (2015)

[15] I. Bunzarov et al., Phys.Part.Nucl.Lett. 11, 404 (2014)

[16] B.B. Mandelbrot, The Fractal Geometry of Nature (Freeman, San Francisco, 1982)

[17] T.G. Dedovich, M.V. Tokarev, Phys. Part. Nucl. Lett. 8, 521 (2011)

[18] T.G. Dedovich, M.V. Tokarev, Phys. Part. Nucl. Lett. 9, 552 (2011)

[19] I. Zborovský, Yu.A. Panebratsev, M.V. Tokarev, G. Škoro, Phys. Rev. D 54, 5548 (1996)

[20] M.V. Tokarev, T.G. Dedovich, Int. J. Mod. Phys. A 15, 3495 (2000)

[21] M.V. Tokarev, T.G. Dedovich, and I.Zborovský, Int. J. Mod. Phys. A 27, 1250115 (2012) 